\title{
ARTICLE
}

\section{TSPO upregulation in bipolar disorder and concomitant downregulation of mitophagic proteins and NLRP3 inflammasome activation}

Giselli Scaini ${ }^{1}$, Tatiana Barichello $\mathbb{1 D}^{1,2}$, Gabriel R. Fries ${ }^{1}$, Elizabeth A. Kennon ${ }^{1}$, Taylor Andrews ${ }^{1}$, Bobby R. Nix ${ }^{3}$, Giovana Zunta-Soares ${ }^{3}$, Samira S. Valvassori ${ }^{4}$, Jair C. Soares ${ }^{3,5}$ and João Quevedo (iD ${ }^{1,3,4,5}$

Bipolar disorder (BD) is a chronic, debilitating illness with a global prevalence of up to $4.8 \%$. The importance of understanding how dysfunctional mitochondria and mitophagy contribute to cell survival and death in BD is becoming increasingly apparent. Therefore, the purpose of this study was to evaluate the mitophagic pathway and NLRP3 inflammasome activation in peripheral blood mononuclear cells (PBMCs) of patients with BD and healthy individuals. Since 18-kDa translocator protein (TSPO) plays an important role in regulating mitochondrial function and since TSPO itself impairs cellular mitophagy, we also investigated the changes in the TSPO-related pathway. Our results showed that patients with BD had lower levels of Parkin, p62/SQSTM1 and LC3A and an upregulation of TSPO pathway proteins (TSPO and VDAC), both in terms of mRNA and protein levels. Additionally, we found a negative correlation between mitophagy-related proteins and TSPO levels, while VDAC correlated negatively with p62/SQSTM1 and LC3 protein levels. Moreover, we found that the gene expression levels of the NLRP3-related proteins NLRP3, ASC, and procasp1 were upregulated in BD patients, followed by an increase in caspase-1 activity as well as IL-1 $\beta$ and IL-18 levels. As expected, there was a strong positive correlation between NLRP3-related inflammasome activation and TSPO-related proteins. The data reported here suggest that TSPO-VDAC complex upregulation in BD patients, the simultaneous downregulation of mitophagic proteins and NLRP3 inflammasome activation could lead to an accumulation of dysfunctional mitochondria, resulting in inflammation and apoptosis. In summary, the findings of this study provide novel evidence that mitochondrial dysfunction measured in peripheral blood is associated with BD.

Neuropsychopharmacology (2019) 44:1291-1299; https://doi.org/10.1038/s41386-018-0293-4

\section{INTRODUCTION}

Bipolar disorder (BD) is a mental health condition that affects approximately $1 \%$ of the population. BD is characterized by unusual shifts in mood, energy, activity levels, and ability to function as well as cognitive impairment $[1,2]$. Acute episodes are associated with cognitive and functional impairments [3], which tend to worsen with the progression of the illness [4-7]. Despite the superior cognitive capacity often present in at-risk individuals [8], patients with a long-term course of illness present with significant impairment in neuropsychological performance compared to early-stage patients and controls $[4,9]$.

Although knowledge regarding the neurobiology of $B D$ is expanding rapidly, and studies have focused on efforts to better characterize the biological basis of this disorder, the precise pathophysiology of $\mathrm{BD}$ remains unknown [10]. BD is a complex pathology that involves several biological pathways, including the interaction of molecular, cellular, and behavioral mechanisms with susceptibility genes, environmental stressors, and biochemical mechanisms [4], leading to inflammation; oxidative stress; mitochondrial and endoplasmic reticulum dysfunction; disrupted apoptotic and neurogenesis pathways [11-14]; and dysregulation of multiple neural systems [15-17]. In recent years, increasing attention has been focused on understanding the relationship between mitochondrial dysfunction and BD. Mitochondrial dysfunction is a characteristic feature of many chronic illnesses that are not currently classified as mitochondrial diseases, such as multiple sclerosis, Parkinson's disease, schizophrenia, depression, autism, and chronic fatigue syndrome [18].

Mitochondria are highly dynamic and act as a central point of the equilibrium between health and disease. In this respect, the adaptation of energy supply to energy demand is central to vital cellular bioenergetic homeostasis and is critically regulated by the dynamics and turnover of the mitochondrial population. A damaged mitochondrion may be able to segregate its damaged components into subcompartments and divide, whereas the healthy mitochondrion with the healthier membrane potential will

\footnotetext{
${ }^{1}$ Translational Psychiatry Program, Department of Psychiatry and Behavioral Sciences, McGovern Medical School, The University of Texas Health Science Center at Houston (UTHealth), Houston, TX, USA; ${ }^{2}$ Experimental Pathophysiology Laboratory, Graduate Program in Health Sciences, University of Southern Santa Catarina (UNESC), Criciúma, SC, Brazil; ${ }^{3}$ Center of Excellence on Mood Disorders, Department of Psychiatry and Behavioral Sciences, McGovern Medical School, The University of Texas Health Science Center at Houston (UTHealth), Houston, TX, USA; ${ }^{4}$ Translational Psychiatry Laboratory, Graduate Program in Health Sciences, University of Southern Santa Catarina (UNESC), Criciúma, SC, Brazil and ${ }^{5}$ Neuroscience Graduate Program, The University of Texas MD Anderson Cancer Center UTHealth Graduate School of Biomedical Sciences, Houston, TX, USA Correspondence: João Quevedo (Joao.L.DeQuevedo@uth.tmc.edu)

These authors contributed equally: Giselli Scaini, Tatiana Barichello
}

Received: 13 August 2018 Revised: 24 October 2018 Accepted: 3 December 2018

Published online: 11 December 2018 
1292

continue to participate in fusion and fission cycles, and the depolarized damaged mitochondrion is often degraded through mitophagy by the PTEN-induced kinase 1 (PINK1)-Parkin pathway [19]. We have previously found downregulation of the mitochondrial fusion-related proteins Mfn-2 and Opa-1 and the upregulation of the fission protein Fis-1 in peripheral blood mononuclear cells (PBMCs) from patients with BD at both gene and protein expression levels [20].

The mitochondrial permeability transition, which is involved in the control of mitochondrial membrane permeabilization, has been suggested to be responsible for the mitophagy of depolarized mitochondria and the induction of apoptosis and necrosis and is mediated by the mitochondrial permeability transition pore (mPTP). The MPTP is composed of a voltagedependent anion channel (VDAC) in the outer membrane, an 18 $\mathrm{kDa}$ translocator protein (TSPO), an adenine nucleotide translocator (ANT), and other proteins [21-23]. TSPO and VDAC mediate various mitochondrial functions, including $\mathrm{Ca}^{2+}$ and ATP flux through the mitochondrial outer membrane; ATP production; reactive oxygen species (ROS) generation; mitochondrial membrane potential $(\Delta \psi \mathrm{m})$ transitions; the control of NADH levels; and inflammation [24-30]. Moreover, the interaction between TSPO and VDAC has been shown to be involved in the autophagic removal of mitochondria by limiting Parkin RBR E3 ubiquitin protein ligase (PARK2)-mediated mitochondrial ubiquitination via the peri-organelle accumulation of ROS [31]. This mechanism could be related to nonphysiological increases in $\mathrm{Ca}^{2+}$ concentration because TSPO deregulates mitochondrial $\mathrm{Ca}^{2+}$ signaling via VDAC and leads to a parallel increase in the cytosolic $\mathrm{Ca}^{2+}$ pools that activate $\mathrm{Ca}^{2+}$-dependent $\mathrm{NADPH}$ oxidase (NOX), thereby increasing ROS [32]. Moreover, converging evidence has shown the role of TSPO and VDAC in NLRP3 inflammasome activation [33-36]. Thus, the TSPO-VDAC complex may be a key mitochondrial pathway providing mitochondria the capability to regulate cell survival and death signals.

The importance of understanding how dysfunctional mitochondria and mitophagy contribute to cell survival and death in BD is becoming increasingly apparent. Therefore, it is important to gain insights into the mechanisms regulating the balance between survival and death and how this process is regulated in cells. Considering our preliminary results that showed that BD patients present an imbalance in mitochondrial fission and fusion towards fission, followed by an increase in the caspase-3 protein levels, our central hypothesis is that the downregulation of mitophagic proteins and the activation of the NLRP3 inflammasome, followed by the upregulation of peripherally detected TSPO and VDAC, may be functionally relevant to clinical outcomes among BD patients. To test this hypothesis, we first examined the relationship between $\mathrm{BD}$ and changes in the mitophagic pathway, NLRP3 inflammasome activation, and the TSPO-related pathway in peripheral blood mononuclear cells (PBMCs) of patients with BD and healthy individuals. Second, we correlated the peripheral data with symptoms to further explore the clinical relevance of our findings.

\section{SUBJECTS AND METHODS}

\section{Subjects}

This study was carried out in accordance with the principles of the Declaration of Helsinki with approval from the Institutional Review Board of the University of Texas Health Science Center at Houston, and written informed consent was obtained from all research participants. Thirty-one participants with type I BD were recruited from the UTHealth Mood Disorders outpatient clinic, and twentyfive healthy controls were recruited from the local community and did not have a psychiatric disorder or family history of major psychiatric or neurological disorders in first-degree relatives. Patients and healthy controls were matched for ethnicity, age, gender and educational level. Participants and healthy controls were assessed with the Mini-International Neuropsychiatric Interview (MINI) to confirm BD diagnosis (in patients) or to exclude a history of psychiatric disorders (in controls) [37]. BD participants were also assessed using the Montgomery-Åsberg Depression Rating Scale (MADRS) [38] and the Young Mania Rating Scale (YMRS) [39] to index the severity of depressive and manic symptoms, respectively. Functioning was assessed with the Global Assessment of Functioning (GAF) Scale and Functioning Assessment Short Test (FAST) $[40,41]$.

\section{Whole-blood sample processing}

Human blood samples were collected in heparin-coated collection tubes. Then, PBMCs were separated using LeucoPREP brand cell separation tubes (Becton Dickinson Labware, Lincoln Park, NJ, USA). PBMC cell pellets were mixed with RPMI-1640 medium containing $10 \%$ DMSO and frozen overnight in a Mr. Frosty brand container with 2-propanol (Nalgene, Rochester, NY) at $-80^{\circ} \mathrm{C}$ following an appropriate postprocessing delay. A previous study in our laboratory established that no differences were found in the predicted frequency of B-lymphocytes, granulocytes, monocytes, natural killer cells, CD4 + T-lymphocytes, or CD8 + T-lymphocytes between $\mathrm{HCs}$ and $\mathrm{BD}$ patients [42]. Moreover, our rationale for using PBMCs as a research tool for this study was based on the following: (1) to perform a follow-up of our previous studies; (2) the availability of PBMCs for clinical interrogation as a noninvasive target that can be monitored serially during the course of the disease, allowing longitudinal studies; and (3) in contrast to postmortem brain tissue that represents the disease endpoint and brain imaging that can only reveal impairments at the neural system level, PBMCs allow us to study dynamic changes involved in mitochondrial dysfunction, as well as allow us to perform correlations between the peripheral mitochondrial function or dysfunction with behavioral, cognitive, and functional parameters.

Gene expression

Total RNA was isolated from PBMCs using an RNeasy Plus Mini Kit (Qiagen, Hilden, Germany) according to the manufacturer's instructions. RNA concentrations were assessed using a NanoDrop (Uniscience, Hialeah, Florida, USA). RNA was converted to CDNA using a high-capacity cDNA reverse transcription (RT) kit (Applied Biosystems, Foster City, California, USA) in a total reaction volume of $10 \mu \mathrm{L}$ containing $2 \mu \mathrm{L}$ of $10 \times$ RT buffer, $0.8 \mu \mathrm{L}$ of $25 \mathrm{x}$ dNMT mix $(100 \mathrm{mM}), 2 \mu \mathrm{L}$ of $10 \mathrm{x} \mathrm{RT}$ random primers, and $1 \mu \mathrm{L}$ of MultiScribe reverse transcriptase ( 50 units $/ \mathrm{mL}$; all reagents were used according to the manufacturer's instructions). Reactions were performed for $10 \mathrm{~min}$ at $25^{\circ} \mathrm{C}, 2 \mathrm{~h}$ at $37^{\circ} \mathrm{C}$, and $5 \mathrm{~s}$ at $85^{\circ} \mathrm{C}$. Subsequently, CDNA was kept at $-20^{\circ} \mathrm{C}$ until it was used for polymerase chain reaction (PCR) amplification. Parkin, Beclin-1, Pink-1, Bnip3, p62/ SQSTM1, LC3, TSPO, VDAC, NLRP3, ASC, and ProCasp1 gene expression levels were measured by real-time quantitative RTPCR using specific TaqMan FAM/MGB assays (Applied Biosystems, assay ID Hs01038322_m1 for Parkin, assay ID Hs01007018_m1 for Beclin-1, assay ID $\mathrm{Hs} 00260868 \mathrm{~m} 1$ for Pink1, assay ID Hs00969291_m1 for Bnip3, assay ID Hs01061917_g1 for p62/ SQSTM1, assay ID Hs01076567_g1 for LC3, assay ID Hs00559362_m1 for TSPO, assay ID Hs01631624_gH for VDAC1, assay ID Hs00918082_m1 for NLRP3, assay ID Hs01547324_gH for ASC, and assay ID Hs00354836_m1 for Casp1). Gene expression values were normalized to eukaryotic 18 S rRNA endogenous controls using a TaqMan VIC/MGB endogenous control inventoried assay (Applied Biosystems, 4319413E). Reactions were performed in an Applied Biosystems 7500 real-time PCR system, which directly detects the PCR product without downstream processing. Reactions were carried out in a total volume of $12 \mu \mathrm{L}$ with $6 \mu \mathrm{L}$ of $2 \mathrm{x}$ TaqMan gene expression master mix (containing ROX, Amplitaq Gold DNA polymerase, AmpErase UNG, dATP, dCTP, dGTP, dUTP, and $\mathrm{MgCl} 2$ ), $0.6 \mu \mathrm{L}$ of 20x TaqMan gene expression 
TSPO upregulation in bipolar disorder and concomitant downregulation of... G Scaini et al.

assay, $0.6 \mu \mathrm{L}$ of $20 \mathrm{x}$ TaqMan endogenous control, $3.8 \mu \mathrm{L}$ of water, and $1 \mu \mathrm{L}$ of cDNA solution. The cycling program consisted of $2 \mathrm{~min}$ at $50^{\circ} \mathrm{C}$ and $10 \mathrm{~min}$ at $95^{\circ} \mathrm{C}$, followed by 40 cycles of $15 \mathrm{~s}$ at $95^{\circ} \mathrm{C}$ and $1 \mathrm{~min}$ at $60^{\circ} \mathrm{C}$. All reactions were performed in triplicate. Relative expression levels were determined by the ddCt method as described by Livak and Schmittgen [43].

\section{Immunoblotting}

To perform the immunoblotting experiments, isolated PBMCs were lysed in $100 \mu \mathrm{L}$ of lysis buffer $(60 \mathrm{mM}$ Tris- $\mathrm{HCl}$ [pH 6.8], $2 \%$ SDS, and $10 \%$ sucrose) supplemented with 1:100 protease inhibitor cocktail (Sigma-Aldrich, St. Louis, MO, USA), followed by sonication and determination of protein concentration using a BCA kit (Thermo Fisher Scientific).

Equal amounts of proteins ( $30 \mu \mathrm{g} /$ well) were fractionated using a 4-20\% precast polyacrylamide gel (Bio-Rad) and electroblotted onto a PVDF membrane using the Trans-Blot Turbo transfer system (Bio-Rad, Hercules, California, USA). Afterwards, the membranes were blocked in Tween-Tris-buffered saline (TTBS: $100 \mathrm{mM}$ Tris- $\mathrm{HCl}[\mathrm{pH} 7.5]$ containing $0.9 \% \mathrm{NaCl}$ and $0.1 \%$ Tween20) containing $5 \%$ nonfat dry milk. The membranes were incubated overnight at $4{ }^{\circ} \mathrm{C}$ with primary antibodies against Parkin (Santa Cruz Biotechnology, Inc., sc32282), p62/SQSTM1 (Cell Signaling Technology Inc., \#8025), LC3A (Abcam, ab52628), TSPO (Abcam, ab109497), and VDAC1 (Abcam, ab14734). Then, the primary antibodies were removed, and the membranes were washed four times for $15 \mathrm{~min}$. After washing, an anti-rabbit or antimouse IgG peroxidase-linked secondary antibody was incubated with the membranes for $1 \mathrm{~h} \quad(1: 10,000$ dilution), and the membranes were washed again. Finally, immunoreactivity was detected using an enhanced chemiluminescence ECL Plus kit (BioRad, Hercules, California, USA). After exposure, the membranes were stripped and incubated with a mouse monoclonal antibody against $\beta$-actin (Sigma-Aldrich, A2228). An anti-mouse IgG peroxidase-linked secondary antibody was incubated with the membranes for $1 \mathrm{~h}(1: 10,000$ dilution), and the membranes were washed again. The signals were visualized using an ECL detection reagent (Bio-Rad) and monitored using a ChemiDoc MP imaging system (Bio-Rad, Hercules, California, USA).

Statistical analysis

Descriptive statistics were used to report sociodemographic and clinical features of the study participants. Categorical variables were compared using chi-squared or Fisher's exact tests. All variables were tested for normal distribution by the Shapiro-Wilk test and histogram visualization. Differences between the two groups (patients vs. controls) were assessed by a Mann-Whitney test or Student's $t$-test for non-normal or normal data distributions, respectively. Correlations between variables were tested with either Pearson's or Spearman's tests depending on their distribution. All statistical tests were two-tailed and used a significance level of $p<0.05$. Statistical analyses were performed using SPSS software version 17.0 (SPSS Inc., Chicago, IL, USA). Binary logistic regression analysis with the elimination of nonsignificant covariates was performed with the diagnosis of $\mathrm{BD}$ as the dependent variable. We considered all variables associated with diagnostic group with $p<0.20$ to be possible confounding factors [44].

\section{RESULTS}

The characteristics of BD participants and healthy controls are shown in Table 1. Notably, in healthy controls, there were no significant differences in age, sex, body mass index, ethnicity, and education between BD participants and healthy controls. The mean YMRS score of the BD group was $4.84 \pm 6.37$, and the mean MADRS score was $12.84 \pm 11.07$. Moreover, a significant difference in functional status, assessed by GAF $(p<0.001)$ and FAST $(p<$
Table 1. Demographic characteristics of all subjects

\begin{tabular}{|c|c|c|c|}
\hline & $\begin{array}{l}\text { Healthy } \\
\text { subjects }\end{array}$ & $\begin{array}{l}\text { Bipolar } \\
\text { subjects }\end{array}$ & $p$-value \\
\hline$n$ & 25 & 31 & \\
\hline Mean age, years & $37.04 \pm 10.21$ & $36.90 \pm 11.95$ & 0.964 \\
\hline Female gender, $n(\%)$ & $18(72 \%)$ & $24(77.4 \%)$ & 0.642 \\
\hline Ethnicity (non-hispanic), $n$ (\%) & $21(84 \%)$ & $28(90.3 \%)$ & 0.477 \\
\hline Education (years), mean \pm SD & $14.74 \pm 1.522$ & $14.05 \pm 2.188$ & 0.265 \\
\hline Body mass index, mean $\pm S D$ & $29.43 \pm 6.64$ & $32.08 \pm 8 / 85$ & 0.229 \\
\hline Smoking, \% & $4.0 \%$ & $31.3 \%$ & 0.010 \\
\hline MADRS, mean $\pm S D$ & NA & $12.84 \pm 11.07$ & \\
\hline YMRS, mean \pm SD & NA & $4.84 \pm 6.37$ & \\
\hline $\mathrm{GAF}$, mean $\pm \mathrm{SD}$ & $90.0 \pm 4.77$ & $56.94 \pm 14.11$ & $<0.001$ \\
\hline FAST, mean \pm SD & $6.76 \pm 9.09$ & $29.78 \pm 14.84$ & $<0.001$ \\
\hline $\begin{array}{l}\text { Number of manic episodes, } \\
\text { mean } \pm \text { SD }\end{array}$ & NA & $21.19 \pm 35.06$ & \\
\hline $\begin{array}{l}\text { Number of hypomanic } \\
\text { episodes, mean } \pm S D\end{array}$ & NA & $22.68 \pm 37.67$ & \\
\hline $\begin{array}{l}\text { Number of depressive } \\
\text { episodes, mean } \pm \text { SD }\end{array}$ & NA & $19.94 \pm 29.61$ & \\
\hline $\begin{array}{l}\text { Number of mixed episodes, } \\
\text { mean } \pm \text { SD }\end{array}$ & NA & $7.57 \pm 26.38$ & \\
\hline $\begin{array}{l}\text { Number of hospitalizations, } \\
\text { mean } \pm \text { SD }\end{array}$ & NA & $3.94 \pm 4.24$ & \\
\hline Illness Duration, mean \pm SD & NA & $10.09 \pm 8.47$ & \\
\hline $\begin{array}{l}\text { Age at first mania episode, } \\
\text { mean } \pm S D\end{array}$ & NA & $21.07 \pm 8.03$ & \\
\hline $\begin{array}{l}\text { Age at first depressive episode, } \\
\text { mean } \pm \text { SD }\end{array}$ & NA & $\begin{array}{l}22.10+ \\
11.70\end{array}$ & \\
\hline \multicolumn{4}{|l|}{ Psychiatric medications (\%) } \\
\hline Lithium & NA & $29 \%$ & \\
\hline Antidepressants & NA & $54.8 \%$ & \\
\hline Atypical antipsychotics & NA & $61.3 \%$ & \\
\hline Typical antipsychotics & NA & $3.2 \%$ & \\
\hline Anticonvulsant & NA & $45.2 \%$ & \\
\hline Benzodiazepines & NA & $12.9 \%$ & \\
\hline \multicolumn{4}{|l|}{ Comorbidities (\%) } \\
\hline GAD & NA & $22.6 \%$ & \\
\hline PTSD & NA & $22.6 \%$ & \\
\hline Social phobia & NA & $9.7 \%$ & \\
\hline PD & NA & $32.3 \%$ & \\
\hline Agoraphobia & NA & $29 \%$ & \\
\hline AUD & NA & $16.1 \%$ & \\
\hline SAD & NA & $56.3 \%$ & \\
\hline
\end{tabular}

0.001 ), was found between patients with $\mathrm{BD}$ and healthy controls. All patients were being treated with various psychiatric medications at conventional doses at the time of the study, including antipsychotics, anticonvulsants, mood stabilizers and antidepressants. Some patients received additional benzodiazepines or stimulants. Nevertheless, a sensitivity analysis of the primary analysis between BD patients under treatment vs. untreated patients was conducted and showed no significant differences in the mRNA and protein expression levels in patients under treatment compared to the levels in untreated patients.

We previously described that BD patients show an imbalance in mitochondrial fission and fusion towards fission, causing 
mitochondrial fragmentation and apoptosis [20]. In line with this observation, we were also interested in evaluating whether the imbalance in mitochondrial dynamics and apoptosis influences the mitophagic pathway. Therefore, we evaluated proteins related to mitophagy in PBMCs from BD patients and healthy controls. Our results showed that patients with $B D$ had lower levels of Parkin, p62/SQSTM1, and LC3A, both in terms of mRNA and protein levels. Parkin, p62/SQSTM1 and LC3A remained significantly decreased in BD patients in comparison with those in controls after controlling for tobacco use (binary logistic regression models). Moreover, there were no significant differences in the gene expression levels of Pink1, Beclin-1 and Bnip3 in PBMCs between healthy controls and subjects with BD (Fig. 1).

Nonetheless, based on previous findings showing that TSPO interacts with VDAC and triggers ROS-mediated inhibition of mitophagy, we investigated the mRNA and protein expression levels of TSPO and VDAC in BD patients and healthy controls. We found that the gene and protein expression levels of TSPO and VDAC were significantly greater in the BD group than levels in the healthy controls (Fig. 2a), and these results remained significantly increased after controlling for tobacco use (binary logistic regression models). Moreover, the ratio of TSPO to VDAC was greater in $\mathrm{BD}$ than that in healthy controls (Fig. 2b). As hypothesized, we found a negative correlation among Parkin, p62/SQSTM1, LC3A and TSPO protein levels, while VDAC was negatively correlated with p62/SQSTM1 and LC3 protein levels; a positive correlation was also found between TSPO and VDCA (Fig. 2c).
Converging evidence has linked NLRP3 inflammasomemediated processes in BD [45] with the role of TSPO in NLRP3 inflammasome activation [35]. In this hypothesis, the overexpression of TSPO activates the NLRP3 inflammasome, which results in IL-1 and IL-18 release by increasing ROS production and calcium dysregulation. Similarly, we found that the gene expression of the NLRP3-related proteins NLRP3, ASC, pro-casp1, and caspase-1 was upregulated in the BD patients compared to that in the healthy controls, followed by an increase in IL-1 $\beta$ and IL-18 expression levels (Supplementary Fig. 1S). After an adjusted analysis, which considered smoking as a covariate and BD as a dependent variable, we found that the previous results remained significant (binary logistic regression models). As expected, there was a strong positive correlation between NLRP3-related inflammasome activation proteins (NLRP3, ASC, caspase-1, IL-1 $\beta$, and IL-18) and TSPO-related proteins (TSPO and VDAC), suggesting that the overexpression of TSPO and VDAC could be one of the mechanisms that activates the NLRP3 inflammasome in BD (Supplementary Fig. 2S).

There are many previous reports proposing that BD can be more comprehensively conceptualized as a condition of multisystemic progression [46-49]. In accordance with this hypothesis, we found that subjects with a more severe functional status had significantly lower levels of mitophagic proteins and higher levels of TSPO, VDAC, NLRP3, ASC, and caspase- 1 activity. Moreover, we also found positive correlations between TSPO, VDAC, NRLP3, ASC, and caspase- 1 expression levels and MADRS and YMRS scores, while mitophagic proteins were negatively correlated with MADRS and YMRS scores (Table 2).
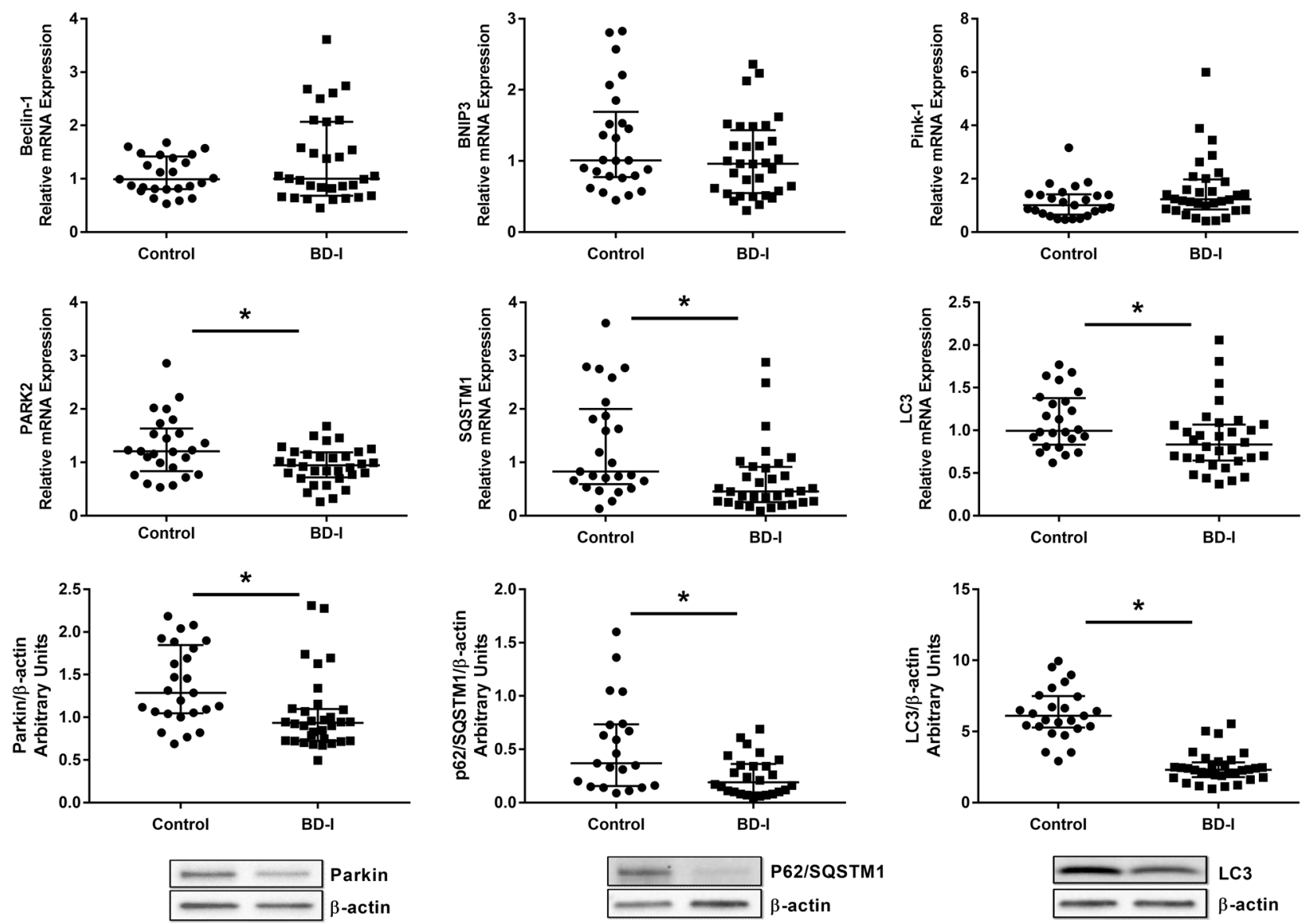

Fig. 1 Relative mRNA and protein expression levels of Pink-1, Parkin, p62/SQSTM1, and LC3 in peripheral blood mononuclear cells (PBMCs) from healthy controls and patients with bipolar disorder type I (BD-I). Data are presented as the median and IQR. Asterisk indicates a significant difference compared to the control group; $p<0.05$ (Mann-Whitney test) 

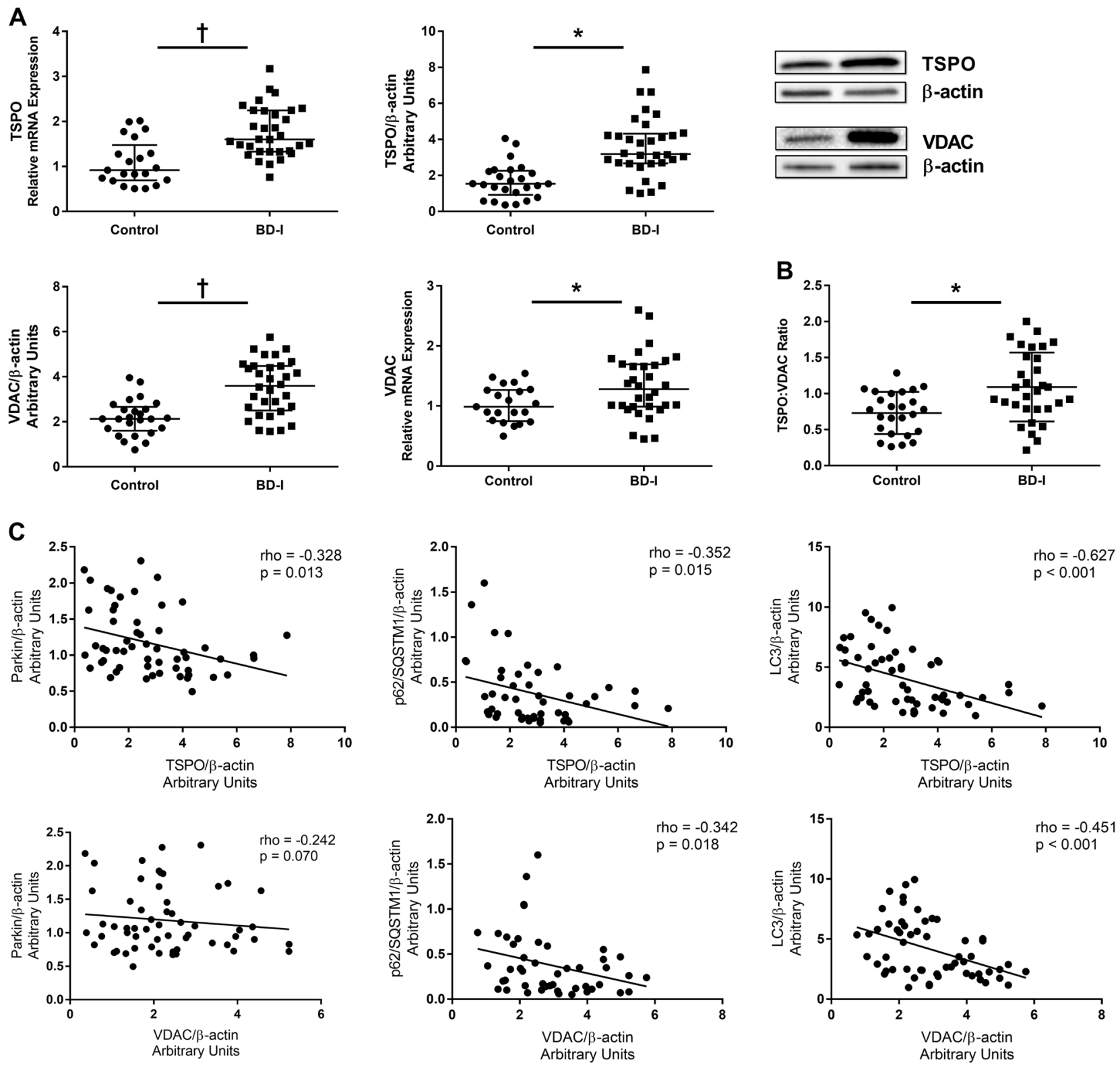

Fig. 2 Relative mRNA expression of TSPO and VDAC (a), TSPO:VDAC ratio (b), and the correlation coefficient between mitophagic protein levels and TSPO and VDAC levels (c) in peripheral blood mononuclear cells (PBMCs) from healthy controls and patients with bipolar disorder type I (BD-I). Data are presented as the median and interquartile range (IQR). Asterisk indicates a significant difference compared to the control group; $p<0.05$ (Mann-Whitney test). Dagger indicates a significant difference compared to the control group; $p<0.05$ (Student's $t$-test). The results were assessed using Spearman's correlation test or Pearson's correlation test, depending on their distribution

\section{DISCUSSION}

Understanding the interface between the adaptation to stress and cell death is important for understanding the pathogenesis of BD. Basal levels of mitophagy are important for maintaining cellular homeostasis and protecting cells against the accumulation of dysfunctional mitochondria [50]. During cellular stress, there is concomitant activation of autophagy/mitophagy and apoptosis, in which enhanced mitophagy is an early response to promote cell survival by removing damaged mitochondria. With overwhelming mitochondrial damage, apoptosis becomes dominant, and inactivation of critical proteins of the autophagy pathway leads to cell death [50], suggesting that there is crosstalk between the mitophagic and apoptotic pathways.

Considering that mitophagy is an essential cellular pathway to eliminate damaged or depolarized mitochondria to maintain the homeostasis of cells and that the dysfunction of mitophagy is closely related to psychiatric disorders, our first aim was to evaluate the mitophagic pathway in PBMCs from BD patients. To the best of our knowledge, we are the first to report changes in proteins related to mitophagy, including Parkin, p62/SQSTM1 and LC3A. A dysregulation of the mitophagic pathway leads to the accumulation of damaged mitochondria, resulting in increased oxidative stress, decreased mitochondrial $\mathrm{Ca}^{2+}$ buffering capacity and loss of ATP, all of which are factors that are particularly harmful in postmitotic cells, such as neurons [51, 52]. Recent research has demonstrated that mitochondrial dynamics are essential to mitophagy since mitochondria are divided into depolarized and polarized mitochondria after fission, and depolarized mitochondria are targeted by mitophagy [53]. Initially, we hypothesized that the downregulation of Mfn2 and Opa1 and the upregulation of Fis 1 in terms of both gene and protein expression levels in BD patients observed in our previous study [20] could 
Table 2. Correlation coefficient between TSPO-related proteins, mitophagy proteins, NLRP3-related inflammasome activation proteins, and clinical data

\begin{tabular}{|c|c|c|c|c|c|c|c|c|c|c|}
\hline & TSPO & VDAC & Parkin & p62 & $\mathrm{LC} 3 \mathrm{~A}$ & NLRP3 & ASC & Casp-1 & IL-1 $\beta$ & IL-18 \\
\hline GAF & $<0.001^{\mathrm{a}}$ & $<0.001^{b}$ & $0.003^{\mathrm{a}}$ & $0.034^{\mathrm{a}}$ & $<0.001^{a}$ & $<0.001^{b}$ & $<0.001^{\mathrm{a}}$ & $<0.001^{\mathrm{a}}$ & $<0.001^{\mathrm{a}}$ & 0.150 \\
\hline FAST & 0.003 & $<0.001^{b}$ & $0.011^{\mathrm{a}}$ & $0.04^{\mathrm{a}}$ & $<0.001^{\mathrm{a}}$ & $0.001^{b}$ & $0.002^{\mathrm{a}}$ & $<0.001^{\mathrm{a}}$ & $<0.001^{\mathrm{a}}$ & 0.174 \\
\hline \multirow[t]{2}{*}{ YMRS } & 0.292 & 0.391 & -0.245 & -0.352 & -0.620 & 0.331 & 0.358 & 0.344 & 0.527 & 0.091 \\
\hline & 0.029 & $0.003^{b}$ & 0.069 & $0.016^{\mathrm{a}}$ & $<0.001^{\mathrm{a}}$ & $0.014^{b}$ & $0.002^{\mathrm{a}}$ & $0.011^{\mathrm{a}}$ & $<0.001^{\mathrm{a}}$ & 0.518 \\
\hline MADRS & $<0.001^{a}$ & $<0.001^{\mathrm{b}}$ & $0.001^{\mathrm{a}}$ & $0.028^{a}$ & $<0.001^{\mathrm{a}}$ & $0.008^{b}$ & $<0.001^{\mathrm{a}}$ & $<0.001^{\mathrm{a}}$ & $<0.001^{a}$ & $0.044^{\mathrm{a}}$ \\
\hline
\end{tabular}

GAF global assessment of functioning, FAST functioning assessment short test, YMRS Young Mania Rating Scale, MADRS Montgomery-Åsberg Depression Rating Scale

aSpearman's correlation test

bearson's correlation test

lead to the activation of mitophagy since the fission process is a prerequisite for mitophagy $[54,55]$; however, we were surprised to observe that BD patients had downregulated mitophagy-related proteins. Despite this, our results corroborate previous findings that showed morphological abnormalities (more mitochondria with a smaller size) in prefrontal cortex neurons of postmortem brains and peripheral cells from BD patients [56]. Taken together, our results and those of previous studies showing that BD patients have impaired oxidative phosphorylation, an overall decrease in energy, and abnormalities in the morphology of mitochondria, followed by apoptosis activation [13, 50], indicate that in BD patients, the number of damaged mitochondria exceeds the capacity of mitophagy, and apoptosis becomes the dominant pathway to minimize tissue damage.

Consistent with these findings, TSPO and VDAC have recently been shown to participate in the ROS-mediated inhibition of mitochondrial quality control, apoptosis and $\mathrm{Ca}^{2+}$ homeostasis. Because these alterations have been reported to contribute to the pathophysiology of BD, our next aim was to evaluate TSPO and VDAC expression levels in BD patients. In this study, we report for the first time that mRNA and protein expression levels of TSPO and VDAC in PBMCs of BD patients are greater than those in healthy controls; an increase in the TSPO:VDAC ratio was also found. Batarseh et al. [57] have shown that the increase in mitochondrial ROS induced by an increase in the TSPO:VDAC ratio could lead to the activation of protein kinase-C $\varepsilon(P K C \varepsilon)$ through the Raf-1-MEK1/2-ERK1/2 pathway, which promotes the gene expression of TSPO via c-Jun, activator protein 1 (AP-1), E-26 oncogene homolog (ETS), and signal transducer and activator of transcription-3 (STAT-3). Likewise, Gatliff et al. [31] hypothesized that TSPO interacts with VDAC, contributing to the efficiency of mitochondrial quality control, which regulates both mitochondrial structure and function. In this study, we showed that when the ratio of TSPO to VDAC increased, mitochondrial production of ATP was limited, and ROS levels increased, which subsequently inhibited PARK2-mediated ubiquitination, P62/SQTM1 recruitment and mitophagy. We have also shown that the TSPO-VDAC complex is responsible for the tangible degeneration of mitochondrial structure and ultrastructure, as observed by an increase in the number of mitochondria, which is an immediate consequence of limited mitophagy in the perinuclear regions of the cell and a decrease in the number of cristae per mitochondria. Similarly, we found a negative correlation between TSPO and VDAC with Parkin and P62/SQSTM1 expression levels, suggesting that subjects with higher expression levels of TSPO and VDAC had lower expression levels of Parkin and P62/SQSTM1, which may be related to a decrease in the mitophagic pathway.
In agreement with a previous report [45], we found that the mRNA expression levels of NLRP3, ASC, procaspase1, and IL-1 $\beta$ were significantly greater in $\mathrm{BD}$ patients than those in healthy controls. Moreover, we were able to show that TSPO and VDAC levels are positively correlated with NLRP3, ASC, caspase- 1, IL-1 $\beta$ and IL-18. Accordingly, previous studies have suggested that TSPO and VDAC are implicated in NLRP3 inflammasome formation. For example, Lee et al. [33] showed that the TSPO ligands Ro5-4864 and PK11195 effectively inhibited ATP-induced NLRP3 inflammasome activation through the prevention of mitochondrial perturbation. Moreover, increased levels of VDAC could lead to the activation of the NLRP3 inflammasome because downregulation of VDAC in THP1 cells results in a considerable decrease in caspase-1 activation and IL-1 $\beta$ secretion after stimulation with various NLRP3 inflammasome activators [35]. Furthermore, as reported previously, the decrease in the mitophagic pathway induced by ROSgenerating mitochondria could lead to spontaneous inflammasome activation [35]. It has been suggested that Beclin-1 and LC3B, which are autophagic proteins required in the initial and late phases of autophagosome formation, act as critical regulators of NLRP3-dependent activation of caspase- 1 and IL-1 $\beta$ and IL-18 secretion. It has also been observed that the loss of mitochondrial integrity is critical for the enhanced activation of caspase-1, both in wild-type macrophages and in autophagy protein-depleted macrophages (Map1/c3b $b^{-/-}$and Becn1 $1^{+/-}$) [34]. Moreover, recent evidence has shown that upon stimulation with LPS, p62, which is a TLR4 ligand that activates NF-KB and primes macrophages for NLRP3 inflammasome activation, is recruited to damaged mitochondria, which promotes mitophagic clearance and prevents excessive inflammasome activation. This suggests that the NF-KBp62/SQSTM1 mitophagic pathway provides an essential regulatory loop that restricts inflammasome activation via elimination of damaged mitochondria [58]. Taken together, it is hypothesized that the upregulation of TSPO and VDAC culminates in an inhibition of the mitophagic pathway, leading to a pronounced accumulation of damaged mitochondria and excessive NLRP3dependent inflammation (Fig. 3). However, this hypothesis must be interpreted with caution because this study was not specifically designed to evaluate the molecular mechanism by which the interaction of TSPO and VDAC leads to a decrease in mitophagic markers and NLRP3 inflammasome activation in BD.

Although mood dysregulation is the primary feature of $B D$, several studies have demonstrated that BD is associated with high rates of illness, which leads to an increase in early mortality rates in $\mathrm{BD}$ patients compared to those in the general population [59]. Thus, there are many previous reports proposing that, in addition to being a neuroprogressive disease [60], BD is more 


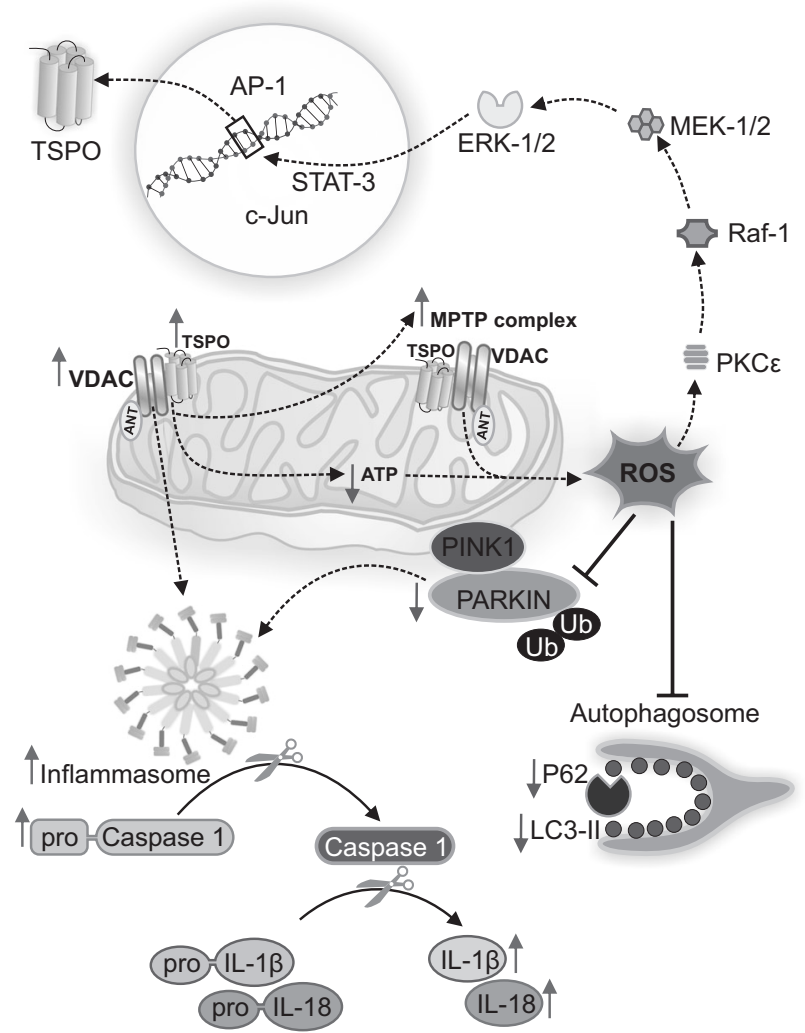

Fig. 3 Potential mechanism of alterations in the TSPO-mediated mitophagic signaling pathway and NLRP3 inflammasome activation in bipolar disorder. Downregulated in PBMCs from BD. Upregulated in PBMCs from BD patients. Mitochondrial ROS induced by an increase in the TSPO:VDAC ratio leads to the activation of protein kinase-C $\varepsilon$ (PKC $\varepsilon$ ) through the Raf-1-MEK1/2-ERK1/2 pathway, thus promoting the gene expression of TSPO via c-Jun, activator protein 1 (AP-1), E-twenty-six oncogene homolog (ETS), and signal transducer and activator of transcription-3 (STAT-3). Moreover, TSPO, via VDAC, triggers an ROS-mediated inhibition of mitophagy, limiting PARK2-dependent ubiquitination and P62/SQTM1 recruitment. Consequently, spontaneous inflammasome activation can be induced by ROS-mediated inhibition of mitophagy. Furthermore, VDAC could lead to the activation of the NLRP3 inflammasome

comprehensively conceptualized as a condition of multisystemic progression $[46,47]$. Our results support this hypothesis since we found correlations between mitophagic proteins, NLRP3 inflammasome components, and TSPO and VDAC expression levels with the severity of manic and depressive symptoms assessed with the YMRS and MADRS scales, respectively. The next set of analyses investigated the correlation of mitochondrial markers measured in this study with functional status evaluated with GAF and FAST. Our study highlighted the significant correlation between decreased levels of mitophagic proteins and increased levels of TSPO, VDAC and NLRP3 inflammasome components with a decrease in functional status. Similar to previous reports [61-63], we found that BD patients present with substantial functional impairments, such as difficulties with activities of daily living, living conditions, occupational/recreational activities, and relationships. Few studies have shown evidence that worsening of functional outcome throughout the course of BD is linked to neuroprogression [6468]. Additionally, Martino et al. [69] a longitudinal study showed that $\mathrm{BD}$ patients with more than five previous affective episodes at baseline exhibited poorer outcomes on all measures of functioning than patients with less than five previous episodes; however, during the follow-up period of the study, the level of functioning remained stable in $\mathrm{BD}$ patients. Despite the fact that the functional outcome is highly variable among individuals with BD [70], difficulties with activities of daily living, living conditions, occupational/recreational activities, and relationships have been associated with increased risks of mortality in general population samples and other patient groups [71-74]. These findings suggest that alterations in the mitochondrial network may play a role in producing psychiatric symptoms and the decline in functional status experienced by these patients. In fact, in the brain, a highly energetic organ that consumes 20 to $25 \%$ of the body's total energy, mitochondria play an important role in many neuronal processes, such as neurodevelopment, neurotransmission, and synaptic plasticity, since such events are spatially and temporally regulated in neurons through bioenergetics, biogenesis, dynamics, and mitochondrial localization [75-77]. Therefore, it is no surprise that compromised mitochondrial function can disrupt neuronal oxidative metabolism and presynaptic calcium signals, and, via regulation of the properties of neurotransmitter release, alter neurotransmission and neuronal growth, two highly energydependent processes, thereby producing symptoms of psychosis and altered mood [78, 79].

We are aware that our research may have some limitations. Methodological limitations to our study include a small sample size, which may be a factor preventing us from observing significant differences in the biomarkers evaluated. Thus, a validation study comprising a larger and more homogeneous genetic sample size is needed to confirm the current findings. Another limitation of this study is its cross-sectional design, which makes it impossible to evaluate the potential utility of mitochondrial markers as characteristic versus state indicators in BD. Moreover, although we did not find any significant effect of medication status in this analysis, TSPO and the mitophagic network could be affected by long-term treatment with psychiatric medications. Finally, the present study only investigated mRNA and protein expression levels of mitochondrial markers and NLRP3 components; therefore, it should be noted that the above mechanism proposed in Fig. 3 is speculative, and further experimental investigations are needed to estimate the functional relevance of TSPO and VDAC to the mitophagic pathway or NLRP3 inflammasome activation in BD patients.

In summary, the findings of this study provide novel evidence that mitochondrial dysfunction measured in peripheral blood is associated with BD. Our findings highlight the importance of further characterization of TSPO and the VDAC pathway in BD patients because changes in the expression of TSPO and VDAC could lead to changes in mitophagic and NLRP3 pathways, resulting in apoptosis and inflammation. Future longitudinal studies focused on the relationship between mitochondrial markers and the progression of BD are necessary as well as an assessment of mitochondrial markers in other tissues of relevance to the pathophysiology of BD.

\section{FUNDING AND DISCLOSURE}

This study was supported in part by grants from the Pat Rutherford, Jr., Endowed Chair in Psychiatry (JCS), the John S. Dunn Foundation (JCS), and the NIMH (R01MH085667-01 A1) (JCS). The Center of Excellence on Mood Disorders and Translational Psychiatry Program (USA) is funded by the Department of Psychiatry and Behavioral Sciences, McGovern Medical School, The University of Texas Medical School at Houston. The Laboratory of Neurosciences (Brazil) is one of the centers of the National Institute for Molecular Medicine (INCT-MM) and one of the members of the Center of Excellence in Applied Neurosciences of Santa Catarina (NENASC). Giselli Scaini thanks the Conselho Nacional de Desenvolvimento Científico e Tecnológico (CNPq) for a postdoctoral fellowship (205680/2014-3). The authors declare no competing interests.

\section{ADDITIONAL INFORMATION}

Supplementary Information accompanies this paper at (https://doi.org/10.1038/ s41386-018-0293-4). 
Publisher's note: Springer Nature remains neutral with regard to jurisdictional claims in published maps and institutional affiliations.

\section{REFERENCES}

1. Chaves OC, Lombardo LE, Bearden CE, Woolsey MD, Martinez DM, Barrett JA, et al. Association of clinical symptoms and neurocognitive performance in bipolar disorder: a longitudinal study. Bipolar Disord. 2011;13:118-23. https://doi.org/ 10.1111/j.1399-5618.2011.00888.x

2. Association AP. Diagnostic and statistical manual of mental disorders. 5th ed. Washington, DC: Author; 2013.

3. Martinez-Aran A, Vieta E, Torrent C, Sanchez-Moreno J, Goikolea JM, Salamero M, et al. Functional outcome in bipolar disorder: the role of clinical and cognitive factors. Bipolar Disord. 2007;9:103-13. https://doi.org/10.1111/j.13995618.2007.00327.x

4. Berk M, Kapczinski F, Andreazza AC, Dean OM, Giorlando F, Maes M, et al. Pathways underlying neuroprogression in bipolar disorder: focus on inflammation, oxidative stress and neurotrophic factors. Neurosci Biobehav Rev. 2011;35:804-17. https://doi.org/10.1016/j.neubiorev.2010.10.001

5. Torres IJ, Boudreau VG, Yatham LN. Neuropsychological functioning in euthymic bipolar disorder: a meta-analysis. Acta Psychiatr Scand Suppl. 2007; 17-26. https://doi.org/10.1111/j.1600-0447.2007.01055.x

6. Rosa AR, Gonzalez-Ortega I, Gonzalez-Pinto A, Echeburua E, Comes M, MartinezAran $A$, et al. One-year psychosocial functioning in patients in the early vs. late stage of bipolar disorder. Acta Psychiatr Scand. 2012;125:335-41. https://doi.org/ 10.1111/j.1600-0447.2011.01830.x

7. Elshahawi HH, Essawi H, Rabie MA, Mansour M, Beshry ZA, Mansour AN. Cognitive functions among euthymic bipolar I patients after a single manic episode versus recurrent episodes. J Affect Disord. 2011;130:180-91. https://doi.org/10.1016/j. jad.2010.10.027

8. Cannon M, Jones P, Gilvarry C, Rifkin L, McKenzie K, Foerster A, et al. Premorbid social functioning in schizophrenia and bipolar disorder: similarities and differences. Am J Psychiatry. 1997;154:1544-50. https://doi.org/10.1176/ ajp.154.11.1544

9. Rosa AR, Magalhaes PV, Czepielewski L, Sulzbach MV, Goi PD, Vieta E, et al. Clinical staging in bipolar disorder: focus on cognition and functioning. J Clin Psychiatry. 2014;75:e450-6. https://doi.org/10.4088/JCP.13m08625

10. Scola G, Kim HK, Young LT, Andreazza AC. A fresh look at complex I in microarray data: clues to understanding disease-specific mitochondrial alterations in bipolar disorder. Biol Psychiatry. 2013;73:e4-5. https://doi.org/10.1016/j.biopsych. 2012.06.028

11. Brown NC, Andreazza AC, Young LT. An updated meta-analysis of oxidative stress markers in bipolar disorder. Psychiatry Res. 2014;218:61-8. https://doi.org/ 10.1016/j.psychres.2014.04.005

12. Gigante AD, Andreazza AC, Lafer B, Yatham LN, Beasley CL, Young LT. Decreased mRNA expression of uncoupling protein 2 , a mitochondrial proton transporter, in post-mortem prefrontal cortex from patients with bipolar disorder and schizophrenia. Neurosci Lett. 2011;505:47-51. https://doi.org/10.1016/j.neulet.2011. 09.064

13. Scaini G, Rezin GT, Carvalho AF, Streck EL, Berk M, Quevedo J. Mitochondrial dysfunction in bipolar disorder: Evidence, pathophysiology and translational implications. Neurosci Biobehav Rev. 2016;68:694-713. https://doi.org/10.1016/j. neubiorev.2016.06.040

14. Konradi C, Sillivan SE, Clay HB. Mitochondria, oligodendrocytes and inflammation in bipolar disorder: evidence from transcriptome studies points to intriguing parallels with multiple sclerosis. Neurobiol Dis. 2012;45:37-47. https://doi.org/ 10.1016/j.nbd.2011.01.025

15. Bielau H, Steiner J, Mawrin C, Trubner K, Brisch R, Meyer-Lotz G, et al. Dysregulation of GABAergic neurotransmission in mood disorders: a postmortem study. Ann N Y Acad Sci. 2007;1096:157-69. https://doi.org/10.1196/annals.1397.081

16. Kishi T, Yoshimura R, Fukuo Y, Okochi T, Matsunaga S, Umene-Nakano W, et al. The serotonin $1 \mathrm{~A}$ receptor gene confer susceptibility to mood disorders: results from an extended meta-analysis of patients with major depression and bipolar disorder. Eur Arch Psychiatry Clin Neurosci. 2013;263:105-18. https://doi.org/ 10.1007/s00406-012-0337-4

17. Rajkowska G. Cell pathology in mood disorders. Semin Clin Neuropsychiatry. 2002; 7:281-92.

18. Morris $G$, Berk M. The many roads to mitochondrial dysfunction in neuroimmune and neuropsychiatric disorders. BMC Med. 2015;13:68 https://doi.org/10.1186/ s12916-015-0310-y

19. Kornmann B. Quality control in mitochondria: use it, break it, fix it, trash it. F1000Prime Rep. 2014;6:15 https://doi.org/10.12703/P6-15

20. Scaini G, Fries GR, Valvassori SS, Zeni CP, Zunta-Soares G, Berk M, et al. Perturbations in the apoptotic pathway and mitochondrial network dynamics in peripheral blood mononuclear cells from bipolar disorder patients. Transl Psychiatry. 2017;7:e1111. https://doi.org/10.1038/tp.2017.83

21. Lemasters JJ, Qian T, He L, Kim JS, Elmore SP, Cascio WE, et al. Role of mitochondrial inner membrane permeabilization in necrotic cell death, apoptosis, and autophagy. Antioxid Redox Signal. 2002;4:769-81. https://doi.org/10.1089/ 152308602760598918

22. Colombini M. VDAC: the channel at the interface between mitochondria and the cytosol. Mol Cell Biochem. 2004;256-257:107-15.

23. Shoshan-Barmatz V, Gincel D. The voltage-dependent anion channel: characterization, modulation, and role in mitochondrial function in cell life and death. Cell Biochem Biophys. 2003;39:279-92. https://doi.org/10.1385/CBB:39:3:279

24. Gatliff J, Campanella M. The $18 \mathrm{kDa}$ translocator protein (TSPO): a new perspective in mitochondrial biology. Curr Mol Med. 2012;12:356-68.

25. Sileikyte J, Blachly-Dyson E, Sewell R, Carpi A, Menabo R, Di Lisa F, et al. Regulation of the mitochondrial permeability transition pore by the outer membrane does not involve the peripheral benzodiazepine receptor (Translocator Protein of 18 kDa (TSPO)). J Biol Chem. 2014;289:13769-81. https://doi.org/10.1074/jbc. M114.549634

26. Veenman L, Gavish M. The role of $18 \mathrm{kDa}$ mitochondrial translocator protein (TSPO) in programmed cell death, and effects of steroids on TSPO expression. Curr Mol Med. 2012;12:398-412.

27. Bono F, Lamarche I, Prabonnaud V, Le Fur G, Herbert JM. Peripheral benzodiazepine receptor agonists exhibit potent antiapoptotic activities. Biochem Biophys Res Commun. 1999;265:457-61. https://doi.org/10.1006/bbrc.1999.1683

28. Hirsch JD, Beyer CF, Malkowitz L, Beer B, Blume AJ. Mitochondrial benzodiazepine receptors mediate inhibition of mitochondrial respiratory control. Mol Pharmacol. 1989;35:157-63.

29. Zisterer DM, Gorman AM, Williams DC, Murphy MP. The effects of the peripheraltype benzodiazepine acceptor ligands, Ro 5-4864 and PK 11195, on mitochondrial respiration. Methods Find Exp Clin Pharmacol. 1992;14:85-90.

30. Papadopoulos V, Fan J, Zirkin B. Translocator protein (18 kDa): an update on its function in steroidogenesis. J Neuroendocrinol. 2018; 30. https://doi.org/10.1111/ jne. 12500

31. Gatliff J, East D, Crosby J, Abeti R, Harvey R, Craigen W, et al. TSPO interacts with VDAC1 and triggers a ROS-mediated inhibition of mitochondrial quality control. Autophagy. 2014;10:2279-96. https://doi.org/10.4161/15548627.2014.991665

32. Gatliff J, East DA, Singh A, Alvarez MS, Frison M, Matic I, et al. A role for TSPO in mitochondrial $\mathrm{Ca}(2+)$ homeostasis and redox stress signaling. Cell Death Dis. 2017;8:e2896. https://doi.org/10.1038/cddis.2017.186

33. Lee JW, Kim LE, Shim HJ, Kim EK, Hwang WC, Min DS, et al. A translocator protein 18 kDa ligand, Ro5-4864, inhibits ATP-induced NLRP3 inflammasome activation. Biochem Biophys Res Commun. 2016;474:587-93. https://doi.org/10.1016/j. bbrc.2016.04.080

34. Nakahira K, Haspel JA, Rathinam VA, Lee SJ, Dolinay T, Lam HC, et al. Autophagy proteins regulate innate immune responses by inhibiting the release of mitochondrial DNA mediated by the NALP3 inflammasome. Nat Immunol. 2011;12:222-30. https://doi.org/10.1038/ni.1980

35. Zhou R, Yazdi AS, Menu P, Tschopp J. A role for mitochondria in NLRP3 inflammasome activation. Nature. 2011;469:221-5. https://doi.org/10.1038/nature09663

36. Menu P, Mayor A, Zhou R, Tardivel A, Ichijo H, Mori K, et al. ER stress activates the NLRP3 inflammasome via an UPR-independent pathway. Cell Death Dis. 2012;3: e261. https://doi.org/10.1038/cddis.2011.132.

37. Sheehan DV, Lecrubier $Y$, Sheehan $\mathrm{KH}$, Amorim $\mathrm{P}$, Janavs J, Weiller $\mathrm{E}$, et al. The Mini-International Neuropsychiatric Interview (M.I.N.I.): the development and validation of a structured diagnostic psychiatric interview for DSM-IV and ICD-10. J Clin Psychiatry. 1998;59:22-33. quiz 4-57

38. Williams JB, Kobak KA. Development and reliability of a structured interview guide for the Montgomery Asberg Depression Rating Scale (SIGMA). Br J Psychiatry. 2008;192:52-8. https://doi.org/10.1192/bjp.bp.106.032532

39. Young RC, Biggs JT, Ziegler VE, Meyer DA. A rating scale for mania: reliability, validity and sensitivity. Br J Psychiatry. 1978;133:429-35.

40. Association AP. Diagnostic and statistical manual of mental disorders, Text Revision (DSM-IV-TR). 4th ed. Washington, DC, USA; 2000.

41. Rosa AR, Sanchez-Moreno J, Martinez-Aran A, Salamero M, Torrent $C$, Reinares M, et al. Validity and reliability of the Functioning Assessment Short Test (FAST) in bipolar disorder. Clin Pract Epidemiol Ment Health. 2007;3:5. https://doi.org/ 10.1186/1745-0179-3-5

42. Fries GR, Bauer IE, Scaini G, Wu MJ, Kazimi IF, Valvassori SS, et al. Accelerated epigenetic aging and mitochondrial DNA copy number in bipolar disorder. Transl Psychiatry. 2017;7:1283. https://doi.org/10.1038/s41398-017-0048-8

43. Livak KJ, Schmittgen TD. Analysis of relative gene expression data using real-time quantitative PCR and the 2(-Delta Delta C(T)) Method. Methods. 2001;25:402-8. https://doi.org/10.1006/meth.2001.1262

44. Victora CG, Huttly SR, Fuchs SC, Olinto MT. The role of conceptual frameworks in epidemiological analysis: a hierarchical approach. Int J Epidemiol. 1997;26:224-7. 
45. Kim HK, Andreazza AC, Elmi N, Chen W, Young LT. Nod-like receptor pyrin containing 3 (NLRP3) in the post-mortem frontal cortex from patients with bipolar disorder: A potential mediator between mitochondria and immune-activation. J Psychiatr Res. 2016;72:43-50. https://doi.org/10.1016/j.jpsychires.2015.10.015

46. Czepielewski L, Daruy Filho L, Brietzke E, Grassi-Oliveira R. Bipolar disorder and metabolic syndrome: a systematic review. Rev Bras Psiquiatr. 2013;35:88-93.

47. Kupfer DJ. The increasing medical burden in bipolar disorder. JAMA. 2005;293:2528-30. https://doi.org/10.1001/jama.293.20.2528

48. Leboyer M, Soreca I, Scott J, Frye M, Henry C, Tamouza R, et al. Can bipolar disorder be viewed as a multi-system inflammatory disease? J Affect Disord. 2012;141:1-10. https://doi.org/10.1016/j.jad.2011.12.049

49. Schloesser RJ, Huang J, Klein PS, Manji HK. Cellular plasticity cascades in the pathophysiology and treatment of bipolar disorder. Neuropsychopharmacology. 2008;33:110-33. https://doi.org/10.1038/sj.npp.1301575

50. Kubli DA, Gustafsson AB. Mitochondria and mitophagy: the yin and yang of cell death control. Circ Res. 2012;111:1208-21. https://doi.org/10.1161/CIRCRESAHA.112.265819

51. Youle RJ, Narendra DP. Mechanisms of mitophagy. Nat Rev Mol Cell Biol. 2011;12:9-14. https://doi.org/10.1038/nrm3028

52. Youle RJ, van der Bliek AM. Mitochondrial fission, fusion, and stress. Science. 2012;337:1062-5. https://doi.org/10.1126/science.1219855

53. Twig G, Shirihai OS. The interplay between mitochondrial dynamics and mitophagy. Antioxid Redox Signal. 2011;14:1939-51. https://doi.org/10.1089/ars.2010.3779

54. Twig G, Elorza A, Molina AJ, Mohamed H, Wikstrom JD, Walzer G, et al. Fission and selective fusion govern mitochondrial segregation and elimination by autophagy. EMBO J. 2008;27:433-46. https://doi.org/10.1038/sj.emboj.7601963

55. Gomes LC, Scorrano L. High levels of Fis1, a pro-fission mitochondrial protein, trigger autophagy. Biochim Biophys Acta. 2008;1777:860-6. https://doi.org/ 10.1016/j.bbabio.2008.05.442

56. Cataldo AM, McPhie DL, Lange NT, Punzell S, Elmiligy S, Ye NZ, et al. Abnormalities in mitochondrial structure in cells from patients with bipolar disorder. Am J Pathol. 2010;177:575-85. https://doi.org/10.2353/ajpath.2010.081068

57. Batarseh A, Li J, Papadopoulos V. Protein kinase C epsilon regulation of translocator protein (18 kDa) Tspo gene expression is mediated through a MAPK pathway targeting STAT3 and c-Jun transcription factors. Biochemistry. 2010;49:4766-78. https://doi.org/10.1021/bi100020e

58. Zhong Z, Umemura A, Sanchez-Lopez E, Liang S, Shalapour S, Wong J, et al. NFkappaB restricts inflammasome activation via elimination of damaged mitochondria. Cell . 2016;164:896-910. https://doi.org/10.1016/j.cell.2015.12.057

59. Crump C, Sundquist K, Winkleby MA, Sundquist J. Comorbidities and mortality in bipolar disorder: a Swedish national cohort study. JAMA. Psychiatry. 2013;70:931-9. https://doi.org/10.1001/jamapsychiatry.2013.1394

60. Passos IC, Mwangi B, Vieta E, Berk M, Kapczinski F. Areas of controversy in neuroprogression in bipolar disorder. Acta Psychiatr Scand. 2016;134:91-103. https://doi.org/10.1111/acps.12581

61. Calabrese JR, Hirschfeld RM, Reed M, Davies MA, Frye MA, Keck PE, et al. Impact of bipolar disorder on a U.S. community sample. J Clin Psychiatry. 2003;64:425-32.

62. Szmulewicz AG, Valerio MP, Lomastro J, Smith JM, Chiappe V, Martino DJ, et al. Neurocognitive functioning in first-episode Bipolar Disorder: Relationship with functional status. J Affect Disord. 2018;228:97-100. https://doi.org/10.1016/j. jad.2017.12.015
63. Rosa AR, Franco C, Martinez-Aran A, Sanchez-Moreno J, Reinares M, Salamero M, et al. Functional impairment in patients with remitted bipolar disorder. Psychother Psychosom. 2008;77:390-2. https://doi.org/10.1159/000151520

64. Berk M. Neuroprogression: pathways to progressive brain changes in bipolar disorder. Int J Neuropsychopharmacol. 2009;12:441-5. https://doi.org/10.1017/ S1461145708009498

65. Berk M, Berk L, Dodd S, Cotton S, Macneil C, Daglas R, et al. Stage managing bipolar disorder. Bipolar Disord. 2014;16:471-7. https://doi.org/10.1111/bdi.12099

66. Berk M, Hallam KT, McGorry PD. The potential utility of a staging model as a course specifier: a bipolar disorder perspective. J Affect Disord. 2007;100:279-81. https://doi.org/10.1016/j.jad.2007.03.007

67. Gama CS, Kunz M, Magalhaes PV, Kapczinski F. Staging and neuroprogression in bipolar disorder: a systematic review of the literature. Rev Bras Psiquiatr. 2013;35:70-4.

68. Kapczinski F, Dias VV, Kauer-Sant'Anna M, Frey BN, Grassi-Oliveira R, Colom F, et al. Clinical implications of a staging model for bipolar disorders. Expert Rev Neurother. 2009;9:957-66. https://doi.org/10.1586/ern.09.31

69. Martino DJ, Igoa A, Scapola M, Marengo E, Samame C, Strejilevich SA. Functional Outcome in the middle course of bipolar disorder: A longitudinal study. J Nerv Ment Dis. 2017;205:203-6. https://doi.org/10.1097/NMD.0000000000000583

70. Reinares M, Papachristou E, Harvey P, Mar Bonnin C, Sanchez-Moreno J, Torrent C, et al. Towards a clinical staging for bipolar disorder: defining patient subtypes based on functional outcome. J Affect Disord. 2013;144:65-71. https://doi.org/ 10.1016/j.jad.2012.06.005

71. Holt-Lunstad J, Smith TB, Layton JB. Social relationships and mortality risk: a meta-analytic review. PLoS Med. 2010;7:e1000316. https://doi.org/10.1371/ journal.pmed.1000316

72. Morrell S, Taylor R, Quine S, Kerr C, Western J. A case-control study of employment status and mortality in a cohort of Australian youth. Soc Sci Med. 1999;49:383-92.

73. Tilvis RS, Laitala $V$, Routasalo $P E$, Pitkala $\mathrm{KH}$. Suffering from loneliness indicates significant mortality risk of older people. J Aging Res. 2011;2011:534781. https:// doi.org/10.4061/2011/534781

74. Ramos LR, Simoes EJ, Albert MS. Dependence in activities of daily living and cognitive impairment strongly predicted mortality in older urban residents in Brazil: a 2-year follow-up. J Am Geriatr Soc. 2001;49:1168-75.

75. Ly CV, Verstreken P. Mitochondria at the synapse. Neuroscientist. 2006;12:291-9. https://doi.org/10.1177/1073858406287661

76. Chang DT, Reynolds IJ. Mitochondrial trafficking and morphology in healthy and injured neurons. Prog Neurobiol. 2006;80:241-68. https://doi.org/10.1016/j pneurobio.2006.09.003

77. Cheng A, Hou Y, Mattson MP. Mitochondria and neuroplasticity. ASN Neuro. 2010;2:e00045. https://doi.org/10.1042/AN20100019

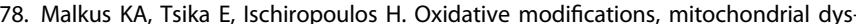
function, and impaired protein degradation in Parkinson's disease: how neurons are lost in the Bermuda triangle. Mol Neurodegener. 2009;4:24. https://doi.org/ 10.1186/1750-1326-4-24

79. McCann RF, Ross DA. So happy together: The storied marriage between mitochondria and the mind. Biol Psychiatry. 2018;83:e47-e9. https://doi.org/10.1016/j. biopsych.2018.03.006 